Proceedings

\title{
Highly Sensitive Electrochemical Glutamate Microsensors for Food Analysis +
}

\author{
Andreas Weltin *, Jochen Kieninger and Gerald A. Urban \\ Laboratory for Sensors, IMTEK-Department of Microsystems Engineering, University of Freiburg, \\ Freiburg, Germany; kieninger@imtek.de (J.K.); urban@imtek.de (G.A.U.) \\ * Correspondence: weltin@imtek.de; Tel.: +49-761-203-7263 \\ + Presented at the Eurosensors 2017 Conference, Paris, France, 3-6 September 2017. \\ Published: 11 August 2017
}

\begin{abstract}
Electrochemical microsensors are ideal to measure substances with low concentration in complex environments. The primary excitatory neurotransmitter L-glutamate is present in many foods as a distinctive flavour (enhancer) with a wide concentration range. In comparison to other methods, electrochemical sensors allow the rapid, precise, cost-effective, online measurement without any sample treatment. We developed a disposable electrochemical microsensor platform with multiple integrated, highly sensitive (detection limit $<150 \mathrm{nM}$ ) and selective enzyme-based glutamate biosensors. We showed both the precise determination of glutamate levels in processed foods with high glutamate content $(15-40 \mathrm{mM})$, e.g., broth, and in foods with low natural concentrations such as different types of cow's milk $(\sim 250 \mu \mathrm{M})$. Hereby, we successfully demonstrated the capabilities of electrochemical biosensors in food monitoring, analysis and quality control.
\end{abstract}

Keywords: monosodium glutamate; neurotransmitter; biosensor; enzyme; food; milk

\section{Introduction}

L-glutamate is well known as the primary excitatory neurotransmitter in the mammalian central nervous system (CNS). It is also widely spread as a distinctive natural flavour in many foods and therefore used as a flavour enhancer. In this context, glutamate is also known under as its sodium salt as which it is often added, monosodium glutamate (MSG). It has a characteristic savoury flavor and adds substantially to the natural flavor of many foods. Glutamic acid as part of the protein molecules is abundant in many protein rich foods. If glutamic acid is released, e.g., due to the denaturing of the proteins in food processing, it dissociates to from the glutamate anion in the neutral $\mathrm{pH}$ range.

Although the relationship between glutamate intake through food and the levels in the CNS is strictly regulated in adults, the determination of glutamate concentrations in food is of interest, not only because glutamate has a multitude of functions outside the CNS [1]. It has to be mentioned however, that glutamate does usually not cross the brain-blood-barrier in adults and is synthesized de novo in the brain for neurotransmission.

Glutamate concentrations in foods can be determined by discontinuous, offline methods such as chemical analyzers or high performance liquid chromatography (HPLC), where time consumption and equipment cost are limitations [2]. Optical (bio-)sensors often lack precision and resolution, especially at low concentrations, as well as a defined zero-point. Enzyme-based electrochemical microsensors allow fast, precise, selective, online measurement from small sample volumes and cost-effective mass fabrication [3]. 


\section{Materials and Methods}

\subsection{Chip and Sensor Fabrication}

Key to reliable electrochemical measurements are appropriate sensitivity and mechanisms for selectivity. We fabricated a disposable multi-sensor platform in a cost-effective hybrid of thin-film and laminate technology (Figure 1). Chip fabrication, including electrode modification and deposition of a $\mathrm{Ag} / \mathrm{AgCl}$ on-chip reference electrode, was done on a wafer-level as described previously $[4,5]$. For sensor integration, glutamate oxidase was stably immobilized on platinum thin-film electrodes by chemical crosslinking into a protein matrix (Figure 1). Besides the enzyme's specificity, a perm-selective layer and the use of a blank sensor guarantee high selectivity.

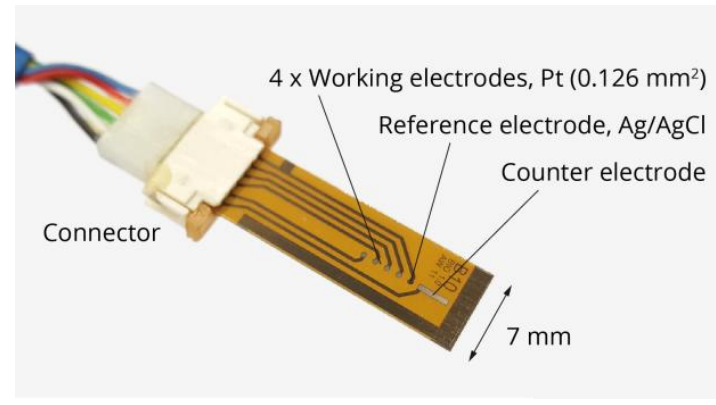

(a)

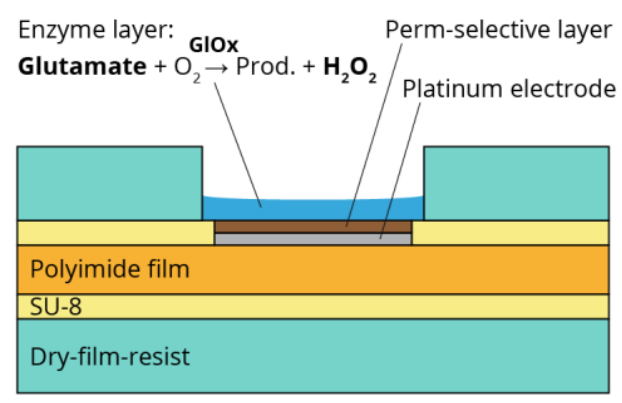

(b)

Figure 1. (a) Photograph of sensor platform including platinum working and counter electrodes and silver/silver chloride reference electrode; (b) Schematic cross-section of thin-film/laminate technology and sensor principle. Glutamate is converted by glutamate oxidase (GlOx) into hydrogen peroxide, which is oxidized at the platinum electrode.

\subsection{Experimental Procedures}

All amperometric electrochemical measurements were carried out in a 3-electrode-setup using on-chip reference and counter electrodes. The working electrodes were polarized to $+450 \mathrm{mV}$ by a multichannel potentiostat. Measurements were performed in $0.1 \mathrm{M}$ phosphate buffered saline (PBS) at $\mathrm{pH} 7.4 \mathrm{~L}$-glutamic acid monosodium salt (Fluka) was used for calibration. Untreated commercial food samples in the microliter range were added to $50 \mathrm{~mL}$ PBS. Instant broth was prepared at $20 \mathrm{~g}$ per litre as recommended.

\section{Results and Discussion}

\subsection{Measurement of High Glutamate Foods}

Instant broth (beef, chicken, vegetable) broth and soy sauce are fermented and processed foods with a typically high glutamate concentration. Figure 2 shows the raw glutamate signals from the addition of different food samples. The online measurement allowed observation of changes in real-time and the complete determination of concentrations within minutes. The obtained measurements and calibrations are highly linear and reproducible. Figure 4 shows the determined glutamate concentrations in different high glutamate foods, which ranged between 15 and $40 \mathrm{mM}$. Reproducibility enabled precise measurements with a relative standard deviation (RSD) around 1\%. The instant broths seem to be adjusted to a certain glutamate level regardless of their flavour. The ingredient yeast extract is very likely the source of the additional glutamate.

\subsection{Measurement of Milk}

High sensitivity ( $\left.>1 \mathrm{nA} \mu \mathrm{M}^{-1} \mathrm{~mm}^{-2}\right)$, low detection limit $(<150 \mathrm{nM})$ and selectivity of the sensor system also allow the direct measurement of foods with two orders of magnitude lower natural concentrations of glutamate such as cow's milk. Different types of commercial (ultra-) 
high-temperature-treated (UHT) whole and skim milk were investigated. First, milk samples were added. Then, glutamate samples for in-line calibration were added in the presence of the milk samples. Both responses show a highly linear and reproducible behavior, which allows a rapid and very precise glutamate measurement (average RSD $<1 \%$ ) (Figure 3). The determined glutamate concentrations in all types of milk were within a close range $(250-300 \mu \mathrm{M})$, but still the different samples of milk could easily be distinguished (Figure 4). The results are summarized in Table 1.

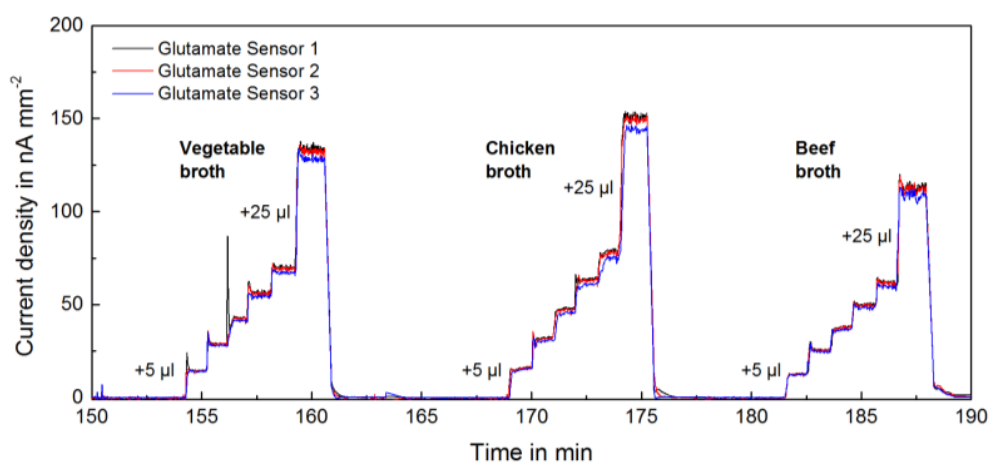

Figure 2. Transient raw signal (blank subtracted) from 3 glutamate sensors upon addition of different types of food samples (instant broth).

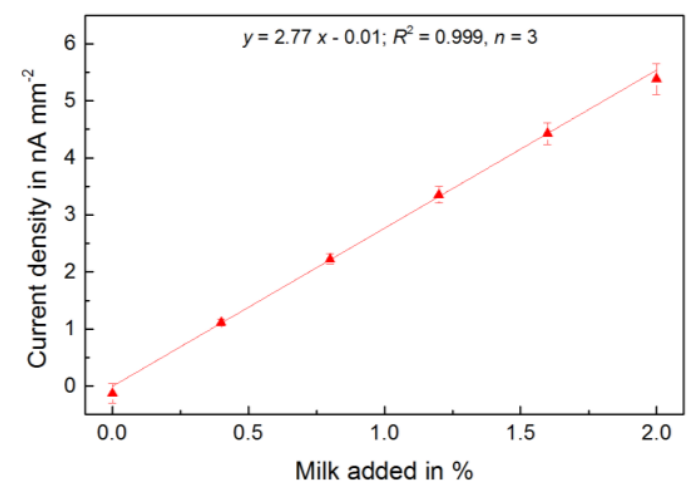

(a)

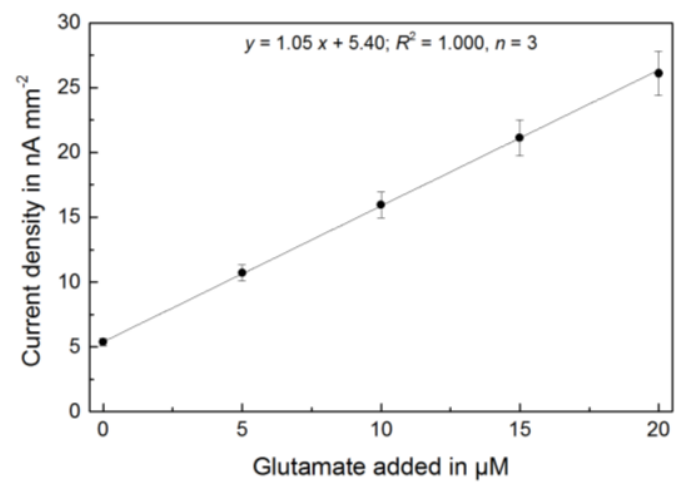

(b)

Figure 3. (a) Glutamate signal measured from successive additions of milk samples to PBS; (b) Subsequent in-line glutamate calibration in the presence of milk showing high linearity.

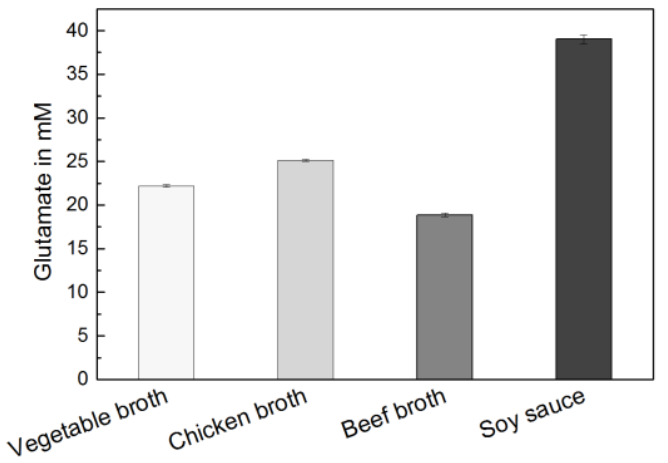

(a)

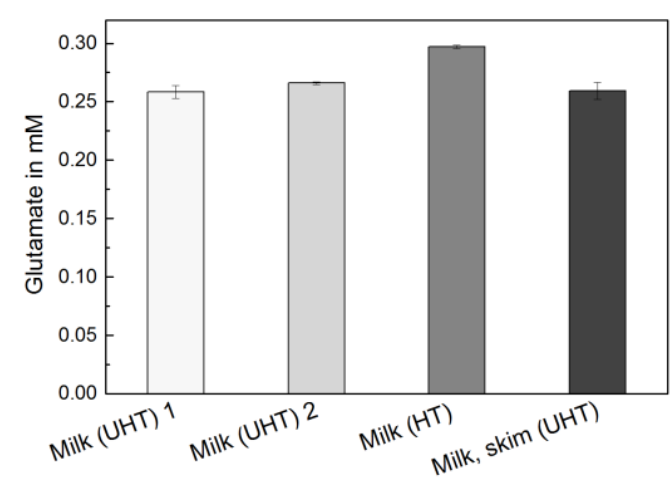

(b)

Figure 4. (a) Determined glutamate concentrations of foods with high glutamate content ( $n=3$ each, average RSD $=1.1 \%$; (b) Determined glutamate concentrations of different types of commercial (ultra-) high-temperature-treated (UHT) cow's milk ( $n=3$ each, average RSD $=0.9 \%$ ). 
Table 1. Glutamate concentrations (incl. standard deviation) measured in different foods.

\begin{tabular}{cc}
\hline Food & Glutamate in $\mathbf{~ m M ~}( \pm$ SD) \\
\hline Vegetable broth & $22.20 \pm 0.16$ \\
Chicken broth & $25.14 \pm 0.14$ \\
Beef broth & $18.86 \pm 0.16$ \\
Soy sauce & $39.00 \pm 0.48$ \\
\hline Milk (UHT) 1 & $0.258 \pm 0.005$ \\
Milk (UHT) 2 & $0.266 \pm 0.001$ \\
Milk (HT) & $0.297 \pm 0.001$ \\
Milk, skim (UHT) & $0.259 \pm 0.007$ \\
\hline
\end{tabular}

\section{Conclusions}

We developed a disposable microsensor platform with multiple integrated, highly sensitive and selective glutamate biosensors. We showed both the precise determination of glutamate levels in processed foods with high glutamate content (15-40 mM), e.g., instant broth and soy sauce, and in foods with low natural concentrations such as different types of cow's milk $(\sim 250 \mu \mathrm{M})$, demonstrating the capabilities of electrochemical biosensors in food monitoring, analysis and quality control.

Conflicts of Interest: The authors declare no conflict of interest.

\section{References}

1. Nedergaard, M.; Takano, T.; Hansen, A.J. Beyond the role of glutamate as a neurotransmitter. Nat. Rev. Neurosci. 2002, 9, 748-755, doi: 10.1038/nrn916.

2. Populin, T.; Moret, S.; Truant, S.; Conte, L.S. A survey on the presence of free glutamic acid in foodstuffs, with and without added monosodium glutamate. Food Chem. 2007, 104, 1712-1717, doi:10.1016/j.foodchem.2007.03.034.

3. Weltin, A.; Kieninger, J.; Urban, G.A. Microfabricated, amperometric, enzyme-based biosensors for in vivo applications. Anal. Bioanal. Chem. 2016, 17, 4503-4521, doi:10.1007/s00216-016-9420-4.

4. Weltin, A.; Kieninger, J.; Enderle, B.; Gellner, A.-K.; Fritsch, B.; Urban, G.A. Polymer-based, flexible glutamate and lactate microsensors for in vivo applications. Biosens. Bioelectron. 2014, 61, 192-199, doi:10.1016/j.bios.2014.05.014.

5. Weltin, A.; Enderle, B.; Kieninger, J.; Urban, G.A. Multiparametric, Flexible Microsensor Platform for Metabolic Monitoring In Vivo. IEEE Sens. J. 2014, 14, 3345-3351, doi: 10.1109/JSEN.2014.2323220.

(c) 2017 by the authors. Licensee MDPI, Basel, Switzerland. This article is an open access article distributed under the terms and conditions of the Creative Commons Attribution (CC BY) license (http://creativecommons.org/licenses/by/4.0/). 